\title{
Finasteride-induced depression: new insights into possible pathomechanisms
}

\author{
Benedikt Römer, MD \& Peter Gass, MD \\ Central Institute of Mental Health, University of Heidelberg, Mannheim, Germany
}

\begin{abstract}
Summary
5-alpha-reductase is involved as a rate-imitating enzyme in the metabolism of steroids. Several 5-alpha-reduced steroids such as dihydrotestosterone, allopregnanolone or tetrahydrocorticosterone have neurotrophic, neuroprotective, and anxiolytic properties. Reduced 5-alpha-reductase activity has been observed during depressive illness in humans. Finasteride inhibits 5-alpha-reductase and can robustly induce anxious and depressive behaviors in rodents. In humans finasteride treatment has been linked to an increase of depressive symptoms. A recent study reported that finasteride treatment inhibits hippocampal neurogenesis in mice. As hippocampal neurogenesis has been linked to emotional behavior, this could be of possible relevance for the pathophysiology of affective disorders. Further studies are needed to evaluate potential neuropsychiatric side effects of finasteride treatment in humans.
\end{abstract}

Keywords: adverse drug reaction, depression, male pattern baldness, neurogenesis

Finasteride is an approved and widely prescribed drug in the treatment of male androgenic alopecia. ${ }^{1}$ Its clinical application is based on the ability to inhibit the type II isoform of the enzyme 5-alpha-reductase. This enzyme is predominantly expressed in human hair follicles and converts testosterone to the biologically more active dihydrotestosterone (DHT). ${ }^{2}$ The finasteride-induced decrease in the bioavailability of DHT attenuates the hormone-dependent progress of hair follicle death and promotes a regrowth of scalp hair. ${ }^{3}$

In addition to catalyzing the reduction in testosterone, 5-alpha-reductase is also involved as a rate-limiting enzyme in the metabolism of other steroid hormones (Fig. 1). A recent study in humans has shown that even the treatment with a daily dose of $5 \mathrm{mg}$ finasteride is sufficient to significantly decrease the concentrations of serum levels of 5-alpha-reduced steroids. ${ }^{4}$ Several of these 5-alpha-reduced steroids are considered as

Correspondence: Benedikt Römer, Psychiatric Division, University Hospital Stavanger, Stavanger, Norway.

E-mail: benediktroemer@hotmail.com

Accepted for publication September 3, 2010 neuroactive steroids, owing to their potent neuromodulatory effects. ${ }^{5,6}$ Neuroactive steroids are also considered as candidate modulators in the pathophysiology and therapy of psychiatric disorders, in particular affective and anxiety disorders. Furthermore, they possess neuroprotective and neurotrophic effects. Neuroactive steroids rapidly alter neuronal excitability by acting at inhibitory GABAA and/or excitatory NMDA receptors. Well studied in this context are the 5a-reduced steroids allopregnanolone and tetrahydrodeoxycorticosterone that have anxiolytic, analgesic, and sedative effects by modulating GABAA receptors. ${ }^{7}$

Both 5-alpha-reductase activity and 5-alpha-reduced steroid concentrations are reduced in depressed patients ${ }^{8}$ and can both be increased by antidepressant (fluoxetine) and atypical antipsychotic (olanzapine) drug treatment. ${ }^{9,10}$ Interestingly, coadministration of finasteride inhibits antidepressant and anxiolytic effects of these drugs, suggesting that collateral effects on neuroactive steroid metabolism are part of their therapeutic efficacy. $^{11}$

In animal studies, inhibition of 5-alpha-reductase leads to behavioral changes with increase in depressive 
5-alpha-reductase

Testosterone

Progesterone

Deoxycorticosterone

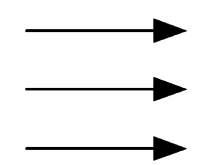

Dihydrotestosterone

Dihydroprogesterone

Dihydrodeoxycorticosterone 3-alpha-hydroxysteroid

dehydrogenase

Figure 1 Finasteride inhibits 5-alpha-reductase as rate-limiting enzyme in the metabolism of steroids. Several 5-alpha-reduced steroids have strong neuromodulatory effects. Reduced 5-alpha-reductase activity has been observed during depressive illness and inhibition of 5-alpha-reductase by finasteride can induce depressive symptoms in animals and humans.

and anxious behaviors. Several reports and one preliminary clinical study suggest that finasteride treatment might induce depressive symptoms in humans. $^{12}$ A recent animal study focussed on the effects of finasteride on neuronal plasticity, a process thought to play a major role in the pathogenesis of and recovery from affective disorders. In this study, finasteride treatment decreased adult neurogenesis in the murine hippocampus. ${ }^{13}$ Adult hippocampal neurogenesis, i.e., the lifelong proliferation of neural precursor cells and their maturation to granule cells which integrate into and modify preexisting neuronal networks, is regarded as a crucial adaptive mechanisms to environmental stimuli. Adult neurogenesis has been shown to play a role in several hippocampus-dependent memory processes, including emotion-associated memories. Thus, the inhibition of hippocampal neurogenesis by finasteride could be an important contributor to changes in central nervous function and emotional behavior. In any case, finasteride has been proven to cause structural changes in a crucial area of the central nervous system, i.e., the limbic system coding for emotional behavior. ${ }^{13}$

Further controlled clinical trials are needed to investigate depressogenic effects of finasteride treatment in humans. However, patients using finasteride should be informed about the potential neuropsychiatric side effects, in particular if taking this drug for cosmetic reasons.

\section{References}

1 Kaufman KD, Olsen EA, Whiting D et al. Finasteride in the treatment of men with androgenetic alopecia. Finasteride male pattern hair loss study group. J Am Acad Dermatol 1998; 39: 578-89.

2 Sudduth SL, Koronkowski MJ. Finasteride: the first 5 alpha-reductase inhibitor. Pharmacotherapy 1993; 13: 309-25; discussion 325-309.
3 Van Neste D, Fuh V, Sanchez-Pedreno P et al. Finasteride increases anagen hair in men with androgenetic alopecia. Br J Dermatol 2000; 143: 804-10.

4 Duskova M, Hill M, Hanus M et al. Finasteride treatment and neuroactive steroid formation. Prague Med Rep 2009; 110: 222-30.

5 Dubrovsky BO. Steroids, neuroactive steroids and neurosteroids in psychopathology. Prog Neuropsychopharmacol Biol Psychiatry 2005; 29: 169-92.

6 Belelli D, Lambert JJ. Neurosteroids: endogenous regulators of the gaba(a) receptor. Nat Rev Neurosci 2005; 6: $565-75$.

7 Lambert JJ, Cooper MA, Simmons RD et al. Neurosteroids: endogenous allosteric modulators of gaba(a) receptors. Psychoneuroendocrinology 2009; 34 (Suppl 1):S48-58. Epub.

8 Uzunova V, Sheline Y, Davis JM et al. Increase in the cerebrospinal fluid content of neurosteroids in patients with unipolar major depression who are receiving fluoxetine or fluvoxamine. Proc Natl Acad Sci USA 1998; 95: 3239-44.

9 Pinna G, Costa E, Guidotti A. SSRIs act as selective brain steroidogenic stimulants (SBSSs) at low doses that are inactive on 5-HT reuptake. Curr Opin Pharmacol 2009; 9: 24-30.

10 Locchi F, Dall'olio R, Gandolfi O, Rimondini R. Olanzapine counteracts stress-induced anxiety-like behavior in rats. Neurosci Lett 2008; 438: 146-9.

11 Finn DA, Beadles-Bohling AS, Beckley EH et al. A new look at the 5alpha-reductase inhibitor finasteride. CNS Drug Rev 2006; 12: 53-76.

12 Rahimi-Ardabili B, Pourandarjani R, Habibollahi P, Mualeki A. Finasteride induced depression: a prospective study. BMC Clin Pharmacol 2006; 6: 7.

13 Römer B, Pfeiffer N, Lewicka S et al. Finasteride treatment inhibits adult hippocampal neurogenesis in male mice. Pharmacopsychiatry 2010; 43: 174-8. 